\title{
Fusiform Gyrus Face Selectivity Relates to Individual Differences in Facial Recognition Ability
}

\author{
Nicholas Furl, Lúcia Garrido, Raymond J. Dolan, Jon Driver, \\ and Bradley Duchaine
}

\begin{abstract}
Regions of the occipital and temporal lobes, including a region in the fusiform gyrus (FG), have been proposed to constitute a "core" visual representation system for faces, in part because they show face selectivity and face repetition suppression. But recent fMRI studies of developmental prosopagnosics (DPs) raise questions about whether these measures relate to face processing skills. Although DPs manifest deficient face processing, most studies to date have not shown unequivocal reductions of functional responses in the proposed core regions. We scanned 15 DPs and 15 non-DP control participants with fMRI while employing factor analysis to derive behavioral components related to face identification or other processes. Repetition suppression specific to facial identities in FG or to expression in FG and STS did not show compelling relationships with face identification ability. However, we identified
\end{abstract}

\section{INTRODUCTION}

Developmental prosopagnosia (DP) is a condition in which individuals (DPs) with otherwise normal vision, intelligence, and social functioning show face recognition impairments in the absence of evident brain injury. Although numerous behavioral studies have enhanced understanding of the cognitive bases of DP (Bentin, Degutis, D'Esposito, \& Robertson, 2007; Humphreys, Avidan, \& Behrmann, 2007; Duchaine \& Nakayama, 2006b; Behrmann, Avidan, Marotta, $\&$ Kimchi, 2005), progress on the neural basis of DP is more limited to date, with the few existing studies of this reviewed below.

An influential account of the functional neuroanatomy of face perception (Haxby, Hoffman, \& Gobbini, 2000) describes a so-called core system in the occipital and temporal lobes. These core regions are considered responsible for visual recognition of facial attributes (Haxby et al., 2000) and are distinguished from a more "extended system," which further processes information resulting from visual recognition. The proposed "core" regions have been associated with face selectivity: they typically respond

University College London robust relationships between face selectivity and face identification ability in FG across our sample for several convergent measures, including voxel-wise statistical parametric mapping, peak face selectivity in individually defined "fusiform face areas" (FFAs), and anatomical extents (cluster sizes) of those FFAs. None of these measures showed associations with behavioral expression or object recognition ability. As a group, DPs had reduced face-selective responses in bilateral FFA when compared with non-DPs. Individual DPs were also more likely than non-DPs to lack expected face-selective activity in core regions. These findings associate individual differences in face processing ability with selectivity in core face processing regions. This confirms that face selectivity can provide a valid marker for neural mechanisms that contribute to face identification ability. more strongly to faces than to nonface objects (cf. Yovel \& Kanwisher, 2005). Neural responses in core regions can also attenuate in response to repeated facial information (i.e., repetition suppression). In normal observers, identity repetition typically suppresses regions in the fusiform gyrus (FG) while repeating more changeable attributes, such as expression, typically leads to BOLD suppression in other regions such as STS (e.g., see Fox, Moon, Iaria, \& Barton, 2009; Rotshtein, Henson, Treves, Driver, \& Dolan, 2005; Andrews \& Ewbank, 2004; Winston, Henson, Fine-Goulden, \& Dolan, 2004).

Although these core face-related brain regions suggest possible loci for neural deficits in DPs, there is scant evidence to date showing functional deficits in these regions. DP case studies using fMRI have mainly reported relatively normal face-selective responses in FG (e.g., see Minnebusch, Suchan, Köster, \& Daum, 2009; Thomas et al., 2009; Behrmann, Avidan, Gao, \& Black, 2007; Williams, Berberovic, \& Mattingley, 2007; Avidan, Hasson, Malach, \& Behrmann, 2005; Hasson, Avidan, Deouell, Bentin, \& Malach, 2003). Despite these numerous "null" (i.e., apparently normal) findings, some atypical findings in individual cases have been identified, including some DPs with no face selectivity (Minnebusch et al., 2009; Bentin et al., 2007; Hadjikhani \& de Gelder, 2002), weakened 
face selectivity (Avidan \& Behrmann, 2009), or expressiondependent FG face selectivity (van de Stock, van de Riet, Righart, \& de Gelder, 2009). One study (using an unusually large sample of DPs) found that voice recognition deficits in DPs were associated with reduced face selectivity in core regions (von Kriegstein et al., 2008). Studies examining repetition suppression have reported apparently normal suppression to repeated (familiar) faces in DPs (Avidan \& Behrmann, 2009; Williams et al., 2007; Avidan et al., 2005).

Thus, functional imaging of DP has provided inconsistent or incomplete evidence for substantially altered neural function in the "core" regions that have been associated with face processing in studies of people with typical face skills. But such evidence might still be reconciled with a role for these core regions in DP if, for example, facerelated effects were reduced or variable in DPs rather than being completely abolished. It remains possible that the magnitude of functional abnormalities might relate systematically to the extent of reduced face skills. Indeed one open possibility, which has not been rigorously investigated to date, is that DPs might constitute the lower tail of a fairly continuous distribution of face identification ability. If so, one would not predict discrete, all-or-nothing functional deficits that should be easily observed in every individual DP. Instead, one would predict functional neuroanatomic correlates of performance might be best detected by testing for continuous relationships between fMRI measures and a wide range of behavioral face identification ability across both DPs and individuals exhibiting normal variation in face skills.

Here, we aimed to implement such an "individual differences" approach by testing not only a sample large enough to allow conventional group contrasts (DPs vs. non-DPs) but also a more systematic analysis of neuroimaging data in relation to the subject-by-subject scores on a battery of tests. We recently applied this approach successfully in a purely structural MRI analysis of 17 DPs and 18 non-DP comparison participants (Garrido et al., 2009). We tested these participants with a battery of behavioral tasks assessing judgments of facial identity, facial expression, and nonface object recognition. Using factor analysis, we showed that we could derive summary scores for orthogonal components of behavioral performance from this battery. Empirically, this yielded separate face identity, face expression, and nonface object behavioral components. In our MRI morphometric analysis, these factor scores showed linear relationships with gray matter volume in temporal cortex across the entire sample (Garrido et al., 2009).

Using the same factor analytic approach to individual differences in behavioral performance, we now extend our analysis of this sample by exploring the relationship of face identification performance deficits to the aforementioned fMRI measures of functional face selectivity and repetition suppression. We hypothesized that the use of these measures may reveal hitherto undiscovered relationships between face identification ability and fMRI signal in the core face-related brain regions, such as FG.

\section{METHODS}

\section{Participants}

Our sample initially included 20 right-handed DPs who contacted the Web site www.faceblind.org and reported severe face recognition difficulties in everyday life. See Garrido et al. (2009) for more information about this participant sample. We confirmed that these participants merited classification as DPs by using the Cambridge Face Memory Test (CFMT in its original form; Duchaine \& Nakayama, 2006a) and the Famous Faces Test (FFT; Duchaine \& Nakayama, 2005). These tests have been validated on DPs in previous studies that also provided the mean CFMT value (mean $\pm S D=57.90 \pm 7.91$ ) from 50 non-DP controls (Duchaine \& Nakayama, 2006a) and the mean FFT value (0.89 \pm 0.09$)$ from 22 non-DP controls (Garrido, Duchaine, \& Nakayama, 2008). We observed that all of the DPs in the present study scored more than two standard deviations below those non-DP means (on both tests). All non-DP participants (see below) in the present study scored better than this criterion for the CFMT and all but two for the FFT. All the DPs showed impaired performance compared with the non-DP averages using a modified $t$ test (Crawford \& Howell, 1998) for single cases. Three DPs were excluded from the study because of neurological or visual abnormality (epilepsy, brain injury, strabismus). The remaining 17 DPs (11 women) were compared with a sample of 18 non-DP participants (11 women), who were matched for age and IQ. Scores on the CFMT and FFT tests in the present study are shown in Figure 1 for the DPs and the non-DP group. The ages of DP and non-DP groups did not significantly differ (mean $\pm S D$ : DPs $=30.9 \pm 7.5$, non-DP $=28.9 \pm 5.7$ ), $t(33)=0.89, p=.38$, nor did IQ (DPs $=123.9 \pm 7.8$, non-DP $=118.9 \pm 8.8), t(33)=1.7, p=.1($ note that IQ

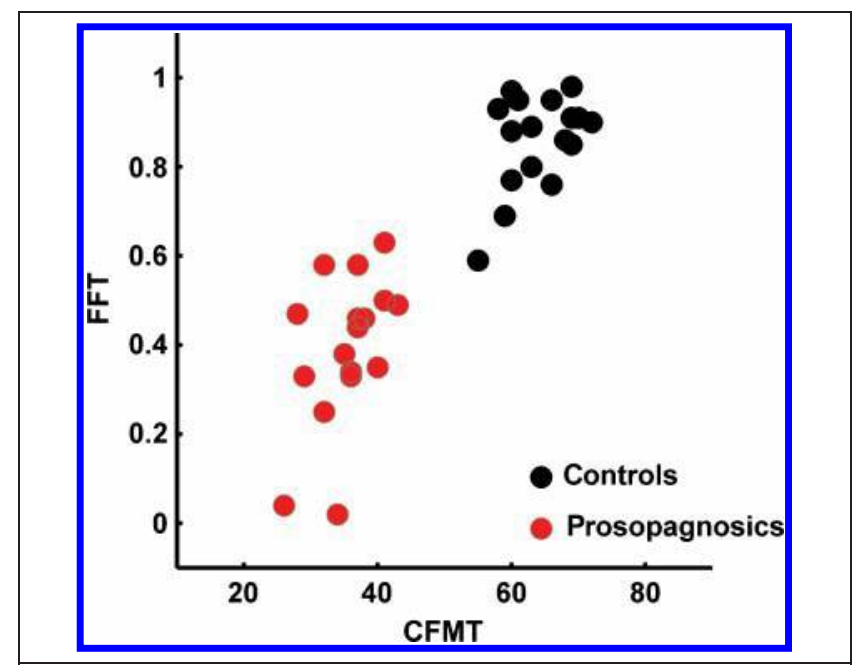

Figure 1. Face identification ability in non-DPs and DPs. Performance of all study participants on the original Cambridge Face Memory Test (CFMT) is indicated on the $x$-axis, and Famous Faces Test (FFT) is indicated on the $y$-axis. Each point represents one participant. We defined some participants as DPs on the basis of performance significantly lower than published non-DP means on both of these two tests. 
measurements were unavailable for two DPs) as measured by Wechsler Abbreviated Scale of Intelligence (PsychCorp, Harcourt Assessment Inc., San Antonio, TX). A neuroradiologist inspected the structural T1- and T2-weighted MRI scans for all these participants and found no evident neurological abnormity in any. All 35 participants showed normal or corrected-to-normal visual acuity as tested with Test Chart 2000 (Thompson Software Solutions, Hatfield, UK). Of these participants, 15 DPs and 15 non-DP controls elected to return for the fMRI experiment. Informed consent was obtained in accordance with procedures approved by The Joint Ethics Committee of The National Hospital for Neurology and Neurosurgery and The Institute of Neurology, London.

\section{Stimuli for fMRI Study}

The stimuli were digitized photographs taken from the KDEF database (Lundqvist, Flykt, \& Öhman, 1998; The Karolinska Directed Emotional Faces, Department of Clini- cal Neuroscience, Psychology Section, Karolinska Institute). For each of four male identities (Figure 2A), we chose photographs acquired on two different days which depicted three viewpoints (left or right three-quarters plus frontal) and four emotional expressions (happy, fearful, neutral, and angry). We converted all photographs to grayscale, cropped the faces to occlude hair and clothing, normalized the images to have luminance distributed with equal mean and range, and then placed them on a gray background. We also digitized photographs of four model classic cars in left or right three-quarters plus frontal views (Figure 2A). These photographs were resized to approximate the size of the face stimuli, and then the same image preparations were implemented as described for the face stimuli.

\section{fMRI Experiment: Design and Procedures}

Throughout the fMRI experiment, participants viewed 15.2-sec blocks, followed by $4 \mathrm{sec}$ of fixation (Figure 2B).
Figure 2. Stimuli and design. (A) Examples of the car stimuli plus facial identities and expressions. (B) The time course of an example block (IdEd condition shown). (C) Participants viewed six types of blocks. Cs $=$ the same car appeared eight times; $\mathrm{Cd}=$ four different cars each appeared twice in random order; IdEd = four identities, and four expressions each appeared twice; IsEd = the same identity appeared throughout, but four expressions each appeared twice; IdEs $=$ four identities each appeared twice, but the expression was the same throughout; IsEs $=$ all faces showed the same identity and expression throughout (but still across different viewpoints, see main text).

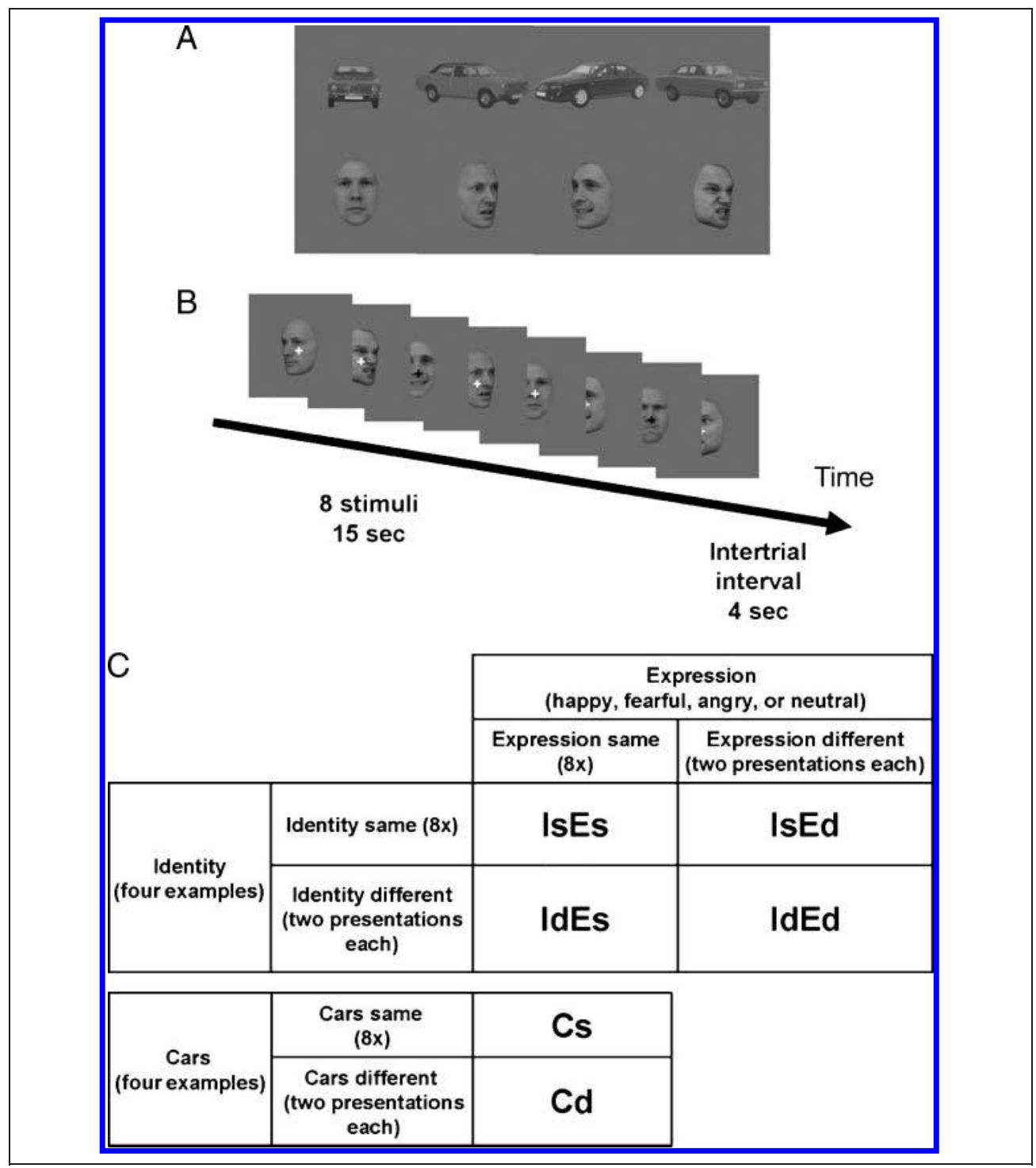


Each block comprised eight successive stimulus presentations, which were $1700 \mathrm{msec}$ each and were preceded by a 200-msec fixation cross. Participants fixated on a cross at the center of the screen (and image) and pressed a key when this cross appeared black on a randomly selected one third of image presentations (otherwise white). Throughout a block, participants viewed images of either all faces or all cars. Each image depicted one of the three views, selected pseudorandomly subject to the constraint that the same view was never immediately repeated (Figure $2 \mathrm{~B}$ ). There were two types of car blocks: In cars-same blocks (Cs), participants viewed eight images of a particular one of the four cars. In cars-different blocks (Cd), participants saw all four cars, each presented twice in random order. There were four types of face blocks (Figure 2C), which conformed to the four cells of a $2 \times 2$ factorial design (Winston et al., 2004). The first factor was Identity repetition: identities could be the same (Is) or different (Id). The second factor was Expression repetition: expressions could be same (Es) or different (Ed). For identity-different/ expression-different blocks (IdEd), each of the four identities and each of the four expressions appeared twice in random order. For identity-same/expression-different blocks (IsEd), all eight images depicted the same identity, with each emotion expressed twice throughout the block. For identity-different/expression-same blocks (IdEs), we presented the four identities twice, always expressing the same emotion. For identity-same/expression-same blocks (IsEs), every image depicted one of the four identities, always expressing one of the four emotions (Figure 2B).
Throughout the experiment, each of these six block types was presented 24 times.

\section{fMRI Data Acquisition}

We used a Siemens Trio 3T system (Siemens, Erlangen, Germany) to acquire T2*-weighted echo-planar functional brain volumes. For each participant, we acquired 430 volumes in each of three scanning sessions. A volume comprised 32 transverse slices aligned parallel to the inferior aspect of the temporal lobes. Images were acquired at a volume repetition time (TR) of $2176 \mathrm{msec}$ with an in-plane resolution of $3 \times 3 \mathrm{~mm}, 2 \mathrm{~mm}$ slice thickness, and $1 \mathrm{~mm}$ slice gap, with echo time $=30 \mathrm{msec}$ and a flip angle of $90^{\circ}$. We discarded the two volumes commencing each session to avoid magnetic equilibrium contamination. In another scanning session (Garrido et al., 2009), T1-weighted MDEFT and T2-weighted structural scans were acquired.

\section{Factor Analysis of Behavioral Test Battery}

We administered a test battery comprising 11 behavioral measures to the 35 participants (17 DPs and 18 non-DPs; see Table 1 and Supplementary Table 1). Each task required recognition of facial identity, facial emotional expression, or nonface objects. These data, for all measures and all participants (i.e., across the entire sample, including both DPs and non-DP participants, in accord with our "individual differences" approach), were submitted to a standard

Table 1. Varimax-rotated Principal Component Weights for Behavioral Tests

\begin{tabular}{|c|c|c|c|c|}
\hline \multirow[b]{2}{*}{ Test } & \multicolumn{3}{|c|}{ Factor } & \multirow[b]{2}{*}{ Reference } \\
\hline & 1 & 2 & 3 & \\
\hline Old/new face identity $\left(\mathrm{A}^{\prime}\right)$ & $0.87^{\mathrm{a}}$ & 0.11 & 0.15 & Duchaine and Nakayama (2005) \\
\hline CFMT (original version) & 0.86 & 0.38 & 0.17 & Duchaine and Nakayama (2006a, 2006b) \\
\hline FFT & 0.80 & 0.34 & 0.25 & Duchaine and Nakayama (2005) \\
\hline Sequential identity matching & 0.76 & 0.16 & 0.13 & Garrido et al. (2009) \\
\hline Cambridge Face Perception & 0.43 & 0.63 & 0.00 & $\begin{array}{l}\text { Duchaine, Germine, and Nakayama (2007), Duchaine, } \\
\text { Yovel, and Nakayama (2007) }\end{array}$ \\
\hline Old/new horses $\left(\mathrm{A}^{\prime}\right)$ & 0.04 & $0.85^{\mathrm{b}}$ & 0.16 & Duchaine and Nakayama (2005) \\
\hline Old/new cars $\left(\mathrm{A}^{\prime}\right)$ & 0.23 & 0.84 & -0.06 & Duchaine and Nakayama (2005) \\
\hline Cambridge hair memory & 0.16 & 0.61 & 0.17 & Garrido et al. (2009) \\
\hline Facial expression films & 0.13 & 0.12 & $0.87^{c}$ & Garrido et al. (2009) \\
\hline Eyes test & 0.13 & 0.21 & 0.80 & Baron-Cohen, Wheelwright, Hill, Raste, and Plumb (2001) \\
\hline Sequential expression matching & 0.39 & -0.15 & 0.49 & Garrido et al. (2009) \\
\hline
\end{tabular}

CFMT $=$ Cambridge face memory test $;$ FFT $=$ Famous faces test .

${ }^{\mathrm{a}}$ Bold items in this column are identity recognition tests.

${ }^{\mathrm{b}}$ Bold items in this column are object recognition tests.

${ }^{\mathrm{c}}$ Bold items in this column are expression recognition tests. 
PCA of the correlation matrix with varimax rotation of the ensuing eigenvector components, as implemented by the SPSS software package for Windows (Rel. 11.0.1. 2001; SPSS Inc., Chicago, IL). We evaluated the first three factors (eigenvalues $>1.0$ ) and computed all participants' factor scores on these three factors. These factor scores were then used as independent variables in regressions including the fMRI data for all participants.

\section{fMRI Preprocessing and SPM First-level Analysis}

We preprocessed and analyzed the fMRI data using SPM5 (Wellcome Trust Centre for Neuroimaging, London; http:// www.fil.ion.ucl.ac.uk/spm/) in conjunction with in-house MATLAB programs (The Mathworks, Natick, MA). Functional scans were realigned, normalized to the standard Montreal Neurological Institute (MNI) echo-planar image template, and then smoothed to an 8-mm FWHM Gaussian kernel in accord with the standard SPM approach.

At the first level of individual subjects, we computed mass univariate time-series models for each participant using proportional scaling, AR(1) autocorrelation modeling, a 1/128-Hz high-pass filter, and regressor convolution with the SPM canonical hemodynamic response model. Covariates of no interest in the design matrix included the six head-motion parameters computed during realignment. Six experimental regressors modeled the onset of each image within the two car and four face block types. We statistically controlled for any correlations between low-level image similarity and block type by adding additional parametric regressors, which modeled the pixel-wise correlation of each presented image and its predecessor (Winston et al., 2004). The first image in each block (which had no predecessor) was assigned the average similarity for the rest of the block for this aspect of the analysis. The mean pixel-wise correlations in each condition were as follows: IdEd $=0.1633$, IsEd $=0.2511$, IdEs $=0.1743$, IsEs $=0.2831, \mathrm{Cd}=0.1639$, $\mathrm{Cs}=0.2059$.

Analysis of the group data then proceeded in two parallel ways. For SPM group analysis, inferences about brain activations were made on the basis of whole-brain statistical parametric maps, using the mass univariate approach and family-wise error (FWE) correcting for multiple corrections at the cluster level using the SPM5 software. This standard SPM approach was thus conducted in a voxel-wise manner. For the ROI group analysis, ROIs were identified in individual participants, with visual confirmation of their anatomical location. Then parameter estimates from the peak of each individually defined ROI were extracted for the various conditions in the main experiment for further analysis in MATLAB (see below). Thus, the ROI analyses differ from the group SPM analysis in considering functionally defined individual ROIs that can fall at somewhat different voxels in different participants, unlike the voxel-wise SPM group analysis. We also analyzed the cluster sizes of particular ROIs (see below).

\section{SPM Group Analysis}

For first-level, fixed-effects analysis, we computed our contrasts of interest for each participant (e.g., face $>$ car selectivity, identity repetition suppression and expression repetition suppression). For our SPM group analysis, wholebrain images of these contrasts were tested statistically using "second level" analyses, which treated participants as a random effect. To test face selectivity and repetition suppression within groups, contrast images were subjected to one-tailed, one-sample $t$ tests. To test whether non-DPs controls differed from DPs, contrast estimates were subjected to two-sample $t$ tests. Finally, these contrasts were tested using linear regressions with the behavioral factor scores. These regressions were carried out by computing the best-fitting line to the data from all participants, when considering the data from all participants (i.e., from both groups), in accord with our "individual differences" approach (for the same approach to structural MR data, see also Garrido et al., 2009).

We first identified clusters of contiguously significant voxels at an uncorrected threshold $(p<.005$, as also used for display purposes in the figures). We then tested these clusters for cluster-level FWE correction $p<.05$ using Gaussian random field theory. We corrected using the whole-brain volume or used conventional small-volume corrections only for regions about which we had a priori hypotheses. To use small volumes conservatively, we required clusterlevel FWE-corrected significance on the basis of both (a) a $10-\mathrm{mm}$ radius sphere surrounding coordinates found in at least one previous fMRI study reporting face-selective or face repetition suppression effects (Fox et al., 2009; Hein \& Knight, 2008; Rotshtein et al., 2005; Andrews \& Ewbank, 2004; Winston et al., 2004; Allison, Puce, \& McCarthy, 2000) and (b) single hemisphere anatomic masks of the posterior fusiform cortex or superior or middle temporal gyrus (for posterior and mid-STS), as derived from the Harvard-Oxford probabilistic atlas available with FSL 4.1 (FMRIB, Oxford, UK; http://www.fmrib.ox.ac.uk/fsl) and thresholded at 25\% of maximum intensity. Exactly the same masks were also used for small volume correction in the structural study of Garrido et al. (2009). For completeness, we additionally tested small volume corrections in the vicinity of the precuneus and posterior cingulate on the basis of a location recently implicated in DP (Avidan \& Behrmann, 2009), but no significant results were obtained using small volumes for these structures. We also allowed small-volume correction of amygdala for contrasts related to existing hypotheses about expression processing. These were implemented on the basis of $10-\mathrm{mm}$ radius spheres surrounding MNI coordinates reported in previous studies of facial expression processing (Das et al., 2005; Vuilleumier, Armony, Driver, \& Dolan, 2003).

\section{Group Analysis of Individual ROIs}

Whereas SPM group analysis provided inference at every voxel-wise location in the brain in the form of a statistical 
parametric map, ROI group analyses provided inference about activity in localized regions as defined in individual participants (which might thus correspond to somewhat different voxels, albeit from similar brain structures, in different participants). The anatomical location of each ROI was confirmed visually with reference to canonical and subject-specific T1-weighted scans in MNI space. Here, we used "FG" to refer in general to neuroanatomic locations within the FG, and we reserved the term "fusiform face area" (FFA) to refer more specifically to just the functionally defined (individually face-selective) ROI located within FG. We identified bilateral FFAs and occipital face areas (OFAs), plus an individual ROI in the right posterior STS, using the contrast all faces $>$ all cars thresholded at $p<1 \times 10^{-4}$ uncorrected. Voxels in these regions fell within the identifiable boundary of just one ROI. For participants who lacked a face-selective area with this criterion, we explored liberal uncorrected significance thresholds (as liberal as $p<.05$ ) to confirm that obvious face-selective areas were not visibly discernable; no areas were found for these cases even with the more lax criteria.

We computed the extent of the ROI cluster sizes for nonDP participants versus DPs. When participants lacked an ROI, the cluster size for that ROI was designated as zero for this analysis. We also sought a measure of individual ROI response independent of cluster size. For this purpose, we extracted beta parameters from first-level fixedeffects models at the peak voxel in each ROI (peak for the functionally defining contrast, e.g., all faces $>$ all cars). Participants who lacked an identifiable face-selective region had no peak voxel and so were excluded from the analysis of individual peaks. The ROI peaks and the cluster sizes were each submitted to two-sample $t$ tests, comparing non-DP participants and DPs. Importantly, we also tested for any relationships these two measures (for ROI peaks and cluster sizes) might have with the behavioral factor scores using linear regression across the entire sample, in accord with our "individual differences" approach. Please note that all our ROIs were defined independently of their relation with the behavioral scores and so did not bias any such relationship.

\section{RESULTS}

\section{Factor Scores for Identity-related Recognition Ability}

Our factor analysis identified three orthogonal factors that were readily interpretable as related to an individual's ability to recognize facial identities, nonface objects, and facial expressions respectively. These results were previously published in Garrido et al. (2009) and so are only briefly summarized in Supplementary Results and Supplementary Table 1. We were especially interested in scores on the first behavioral factor, which provide a summary measure of facial identification ability that is necessarily orthogonal to the object- and expression-related factors. We ran linear regression analyses comparing the scores derived from these factors with fMRI responses in the whole sample (both non-DPs and DPs considered together, in accord with our "individual-differences" approach, as described later). For completeness, in the Supplementary Results, we considered any relations of the fMRI data to the behavioral factors within one or other group (DP or nonDP) considered alone, although that approach inevitably has less power.

\section{Face Selectivity: SPM Group Analysis}

We began by searching every voxel in the whole brain for face selectivity (i.e., we tested the contrast: all faces $>$ all cars) at $p<.05$ FWE-corrected cluster level. We report whole-brain corrected effects or small volume corrections where applicable. All participants were included in the SPM group analyses, regardless of whether they also expressed individually defined ROIs.

For non-DP participants, we observed face-selective peaks in regions of bilateral FG, bilateral posterior STS, right mid-STS, anterior temporal cortex, and bilateral amygdala, with significant effects also spreading into bilateral medial-temporal cortex (Figure 3 and Table 2). When we computed the same contrast for the DP participants (Figure 3 and Table 2), substantially fewer voxels were visible:

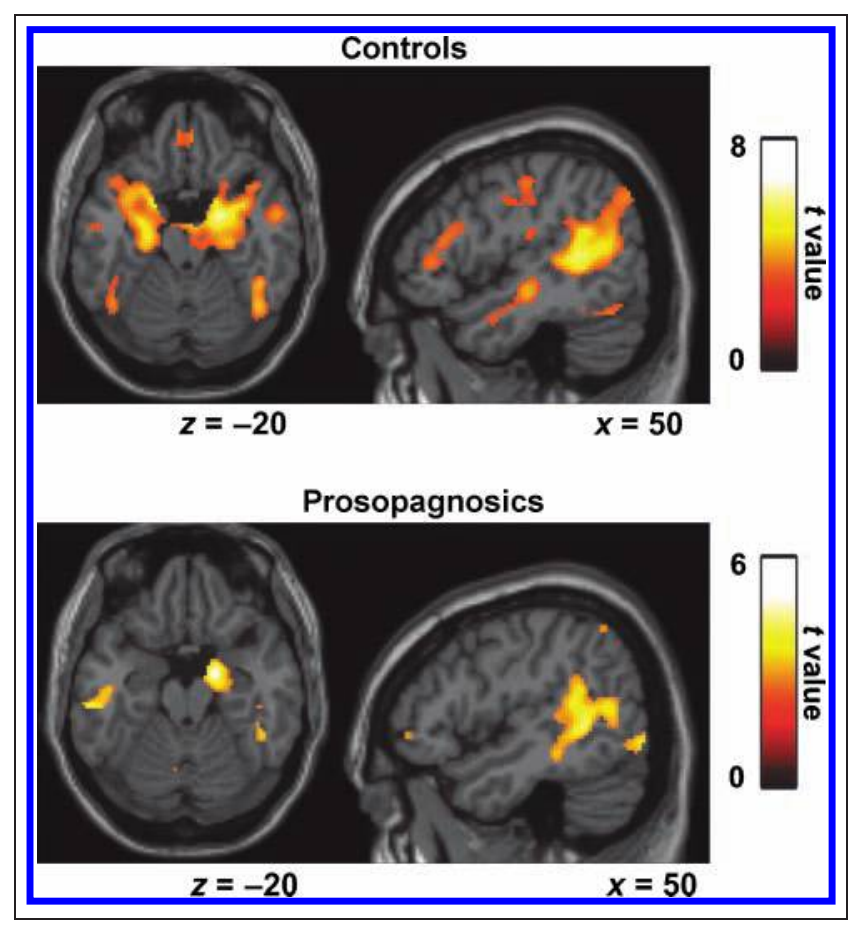

Figure 3. SPM random-effects analysis of face selectivity. Regions in stereotactic space showing significant differences between all faces and all cars in non-DP controls (top) and DPs (bottom) include right and left FG and right posterior and mid-STS. The right hemisphere is shown on the right-hand side of the transverse images. All clusters $p(\mathrm{FWE})<.05$ at the cluster level, whole brain corrected, except left FG (small volume corrected). 
Table 2. Linear Relationships between Face Selectivity and Behavioral Factor Scores

\begin{tabular}{|c|c|c|c|}
\hline & Peak MNI ( $x$ y z $z)$ & z Score & Cluster Size (Voxels) \\
\hline \multicolumn{4}{|l|}{ Non-DPs: All Faces > All Cars } \\
\hline Right STS ${ }^{\mathrm{a}}$ & $54-428$ & 5.87 & 2464 \\
\hline Bilateral amygdalae, medial-temporal cortices and hippocampi ${ }^{\mathrm{a}}$ & $20-4-18$ & 5.72 & 6821 \\
\hline Right $\mathrm{FG}^{\mathrm{a}}$ & $46-48-24$ & 5.07 & 298 \\
\hline Left STS ${ }^{\mathrm{a}}$ & $-56-4612$ & 4.72 & 1692 \\
\hline Left $\mathrm{FG}^{\mathrm{b}}$ & $46-50-28$ & 3.99 & 213 \\
\hline \multicolumn{4}{|l|}{ DPs: All Faces > All Cars } \\
\hline Right amygdala ${ }^{a}$ & $18-6-20$ & 4.96 & 418 \\
\hline Right STS, middle temporal gyrus and $\mathrm{FG}^{\mathrm{a}}$ & $54-4414$ & 4.53 & 1575 \\
\hline \multicolumn{4}{|l|}{ Positive Linear Associations with Identification Factor (1) } \\
\hline Left temporal pole ${ }^{a}$ & $-3418-28$ & 5.14 & 299 \\
\hline Right $\mathrm{FG}^{\mathrm{b}}$ & $46-62-18$ & 3.55 & 98 \\
\hline Left $\mathrm{FG}^{\mathrm{b}}$ & $-48-58-28$ & 4.26 & 193 \\
\hline \multicolumn{4}{|l|}{ Positive Linear Associations with Expression-related Factor (3) } \\
\hline Right visual cortex ${ }^{a}$ & $12-8820$ & 4.74 & 237 \\
\hline Right amygdala/uncus ${ }^{\mathrm{b}}$ & $220-32$ & 4.53 & 1575 \\
\hline
\end{tabular}

$p($ FWE $)<.05$ cluster-level corrected.

${ }^{\mathrm{a}}$ Whole brain corrected.

${ }^{\mathrm{b}}$ Small volume corrected.

1,993 voxels in DP clusters, but 11,488 voxels in non-DP clusters. For DPs, one cluster peaked in right amygdala and spread into right medial-temporal cortex, whereas another peaked in right posterior STS and spread inferiorly into the right FG. In DPs, anterior temporal cortex and left FG showed no significantly face-selective voxels. Despite the visible differences in the significant statistical parametric maps for the two groups considered separately, when we contrasted non-DPs and DPs directly using a one-tailed two-sample $t$ test, no regions were significant at $p(\mathrm{FWE})<.05$, using either whole brain or small volume correction.

We nevertheless observed significant effects of face recognition ability when using linear regression to test for quantitative relationships between face selectivity in fMRI and the identity-related factor scores as measured behaviorally (Figure 4 and Table 2) across the entire sample in accord with our "individual differences" approach. Notably, we found such effects in bilateral FG, within the distribution of face-selective effects observed in non-DP participants. A region near the left temporal pole was also significant at $p(\mathrm{FWE})<.05$ cluster level using whole-brain correction (Figure 4 and Table 2 ). In all three regions (right and left FG and left temporal pole), better face identifi- cation factor scores were associated with increased face selectivity.

We also tested for significant linear relationships with the second and third behavioral factors. As the second and third factors were derived to be orthogonal to the first, they reflect noncorrelated abilities in our participants. We observed no significant interactions between the regressor for first factor and those of the other two factors. Moreover, the regressor for the second (object-related) factor showed no significant linear relationship. For the third (expression-related) factor, across the whole sample, we observed correlations with face selectivity in a region bordering the right posterior amygdala and uncus (small volume corrected for the right amygdala) and a region (whole brain corrected) of early visual cortex (Figure 4 and Table 2).

In sum, we found robust correlations between face identification ability across our entire sample and face selectivity in FG and anterior temporal cortex; but we found no comparable relationship when considering the other factor scores. The lack of a relationship with FG for the third factor seems unlikely to reflect a simple lack of statistical power, as linear relationships were detected outside of FG and temporal pole (in visual cortex and amygdala) for that factor. 


\section{Identification of Individual ROIs}

We also assessed face-selective ROIs for every individual participant. As in previous studies (Minnebusch et al., 2009; Bentin et al., 2007; Williams et al., 2007; Avidan et al., 2005; Hasson et al., 2003; Hadjikhani \& de Gelder, 2002), most DPs evinced right FFAs. There were three exceptions (Figure 5 and Supplementary Table 3): one DP lacked any reliable right ventral temporal face selectivity (hence obtained an ROI cluster size of zero for this). Two additional DPs showed right temporal lobe face selectivity that was diffuse and not divided into distinct regions (even at more conservative thresholds), so we eliminated those two DPs from further ROI group analysis. The absence of left FFA was more common. Five DPs and two non-DPs lacked left FFAs. Eight DPs and three non-DPs lacked any reliable face-selective activation within the right STS. One of the aforementioned DPs with diffuse right temporal lobe face selectivity had no separable region in right posterior STS and so was excluded from ROI group analysis of right posterior STS. We note that posterior STS face selectivity was not likely to be missed in these DPs because of our choice of threshold. Indeed, most DPs lacking STS selectivity nevertheless showed some abnormal face selectivity in the form of a nearby cluster located near V5, which was visibly outside the STS. As we have no unequivocal evidence suggesting this region might be homologous to posterior STS as observed in non-DP participants, we did not classify it as STS. The presence or absence of clearly defined OFA was not associated with DP. Five DPs and six non-DPs lacked right OFA, whereas nine DPs and seven non-DPs lacked left OFA.

We note that the operational definition of an ROI depended on the choice of significance threshold. To avoid false positives, we could have relied on conservative thresholds that were multiple comparison corrected at the first level (e.g., see Avidan \& Behrmann, 2009) or required responses to faces to be numerically at least twice that of responses to nonface objects (cf. Yovel \& Kanwisher, 2005). However, these more conservative criteria inevitably increase the tendency to "miss" effects that might be apparent at less stringent uncorrected thresholds. Accordingly, for purely descriptive purposes, we also explored more liberal uncorrected significance thresholds such as $p<.01$ or $p<$ .05 to examine if any trend for a particular ROI to emerge would then become apparent in an individual who did not show an ROI at our preselected more stringent threshold.
However, at such lowered thresholds, many participants showed first-level results contaminated by uniformly distributed background noise permeating the whole brain, from which it proved problematic to discern any weakly significant ROIs. We therefore optimized our operational definition by selecting an uncorrected significance level that offered unambiguously interpretable ROIs (for at least some of the core areas) in every participant, relative to the presence of background noise and obvious false-positives. We note also that although our ROI selection procedure necessarily had led to the exclusion of some participants from the ROI group analysis (Figure 5 and Supplementary Table 3), no participants were excluded from the SPM group analysis. We thus sought to avoid any potential weaknesses associated with either individual ROI or group SPM approaches alone by demonstrating key findings that were convergent across both approaches.

To summarize, all participants were included in the SPM group analysis. All participants were also included in the ROI group analysis of cluster sizes, except for the two participants with undifferentiated face selectivity (see above). For the analysis of individual ROI peaks, participants were excluded from analysis only if they failed to clearly manifest the relevant ROI. The excluded participants are shown in black in the matrix shown in Figure 5 and listed as "no ROI" in Supplementary Table 3.

\section{Face Selectivity and Cluster Size: Group Analysis of Individual ROIs}

The matrix in Figure 5 shows the peak difference between faces and cars for each ROI in each participant. Rows are sorted within group by the identity-related behavioral factor. Rows filled in black depict the participants mentioned above who did not show any identifiable ROI and were therefore excluded from further analyses of ROI peaks. From this matrix, a positive association is evident between the identity-related factor scores and face selectivity at the ROI peaks. These visual observations were confirmed statistically as follows.

Using one-tailed, two-sample $t$ tests, we found significant differences in peak face selectivity (faces $>$ cars) between non-DPs and DPs for the FFA in the right hemisphere, $t(25)=2.62, p=.007$, and left hemisphere, $t(21)=2.29$, $p=.016$ (Figure 7). In agreement with the SPM group analysis (which had been in voxel-wise stereotactic space,

Figure 4. Behavioral factor scores in relation to brain face selectivity: SPM random-effects analysis. (A) Linear relationships with identity-related behavioral factor scores, across the entire sample (including both DP and non-DP participants) emerge in the right and left FG (both $p$ (FWE) $<$ .05 , cluster level, small volume corrected) and anterior temporal cortex $(p(\mathrm{FWE})<.05$, cluster level, whole brain corrected). The right hemisphere is shown on the right-hand side. (B) The contrast (difference in beta parameters) for all faces $>$ all cars (computed for each participant at the fixed-effects level and grand mean scaled to zero) is plotted as a function of the identity-related factor scores, with each point corresponding to one individual participant. The regression lines (fit to all participants) are shown in blue. (C) Right visual cortex ( $p$ (FWE) $<.05$, cluster level, whole brain corrected) and amygdala $(p(\mathrm{FWE})<.05$, cluster level, small volume corrected) regions showing relationships with expression-related behavioral factor scores. The right hemisphere is shown on the right-hand side. (D) The contrast all faces $>$ all cars from the peak voxels in right visual cortex and amygdala plotted as a function of expression-related factor score. The regression lines (fit to the whole sample) are shown in blue. Note all participants were included in SPM analyses. 
rather than for individually defined ROIs as now), we observed a significantly positive linear relationship between the peak face selectivity and the identification factor scores for FFA (Figure 5) on the right, $\beta=0.41, t(25)=2.89$, $p=.004$, and left, $\beta=0.28, t(21)=2.36, p=.015$. These significant effects were complemented by significant correlations with behavioral Factor 1 (when partialling out Factors 2 and 3$)$ in right FFA ( $\rho=0.52, p=.004)$ and left FFA ( $\rho=0.48, p=.015)$. We also analyzed whether the functional size (volume) of face-selective ROIs (measured as

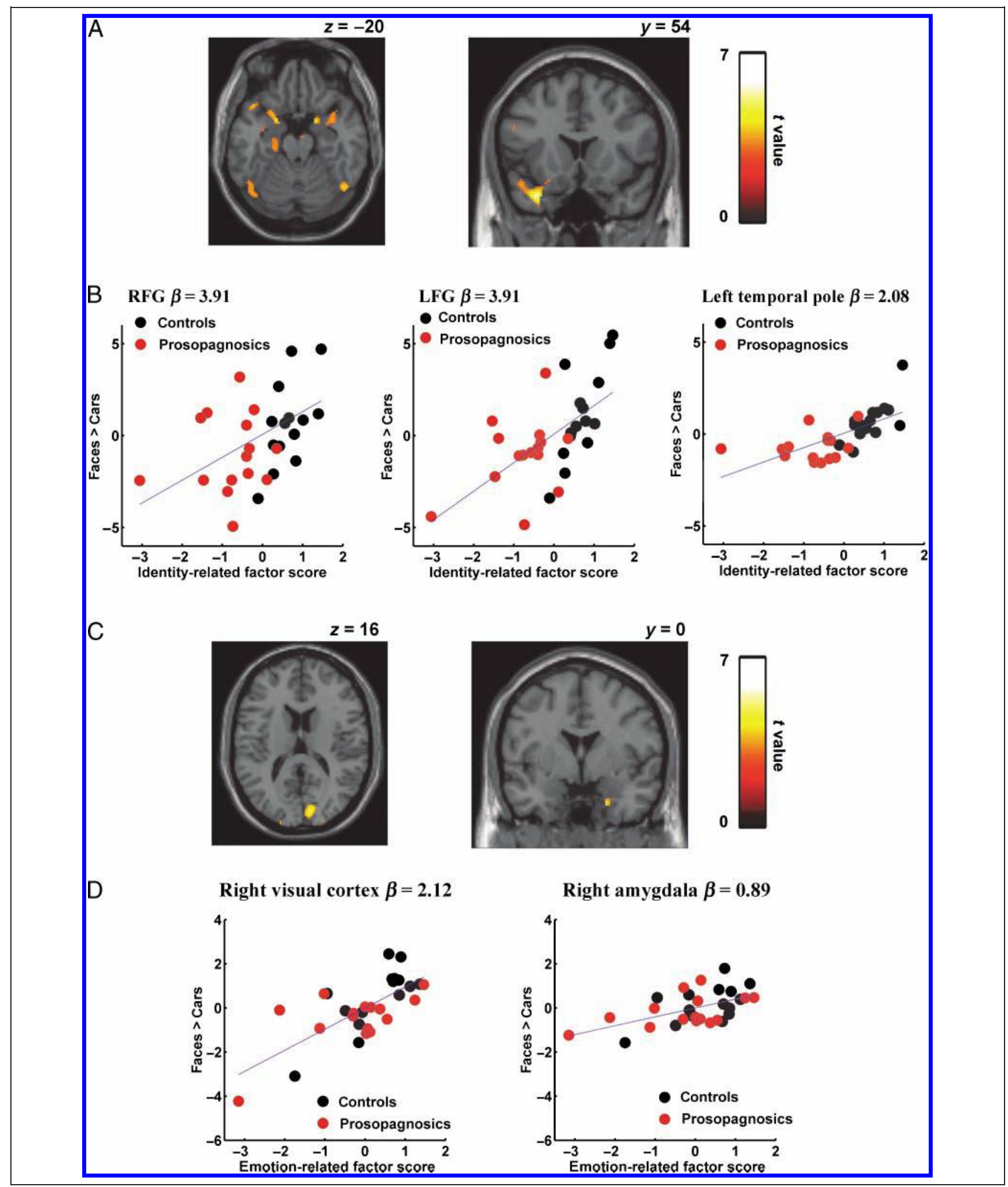




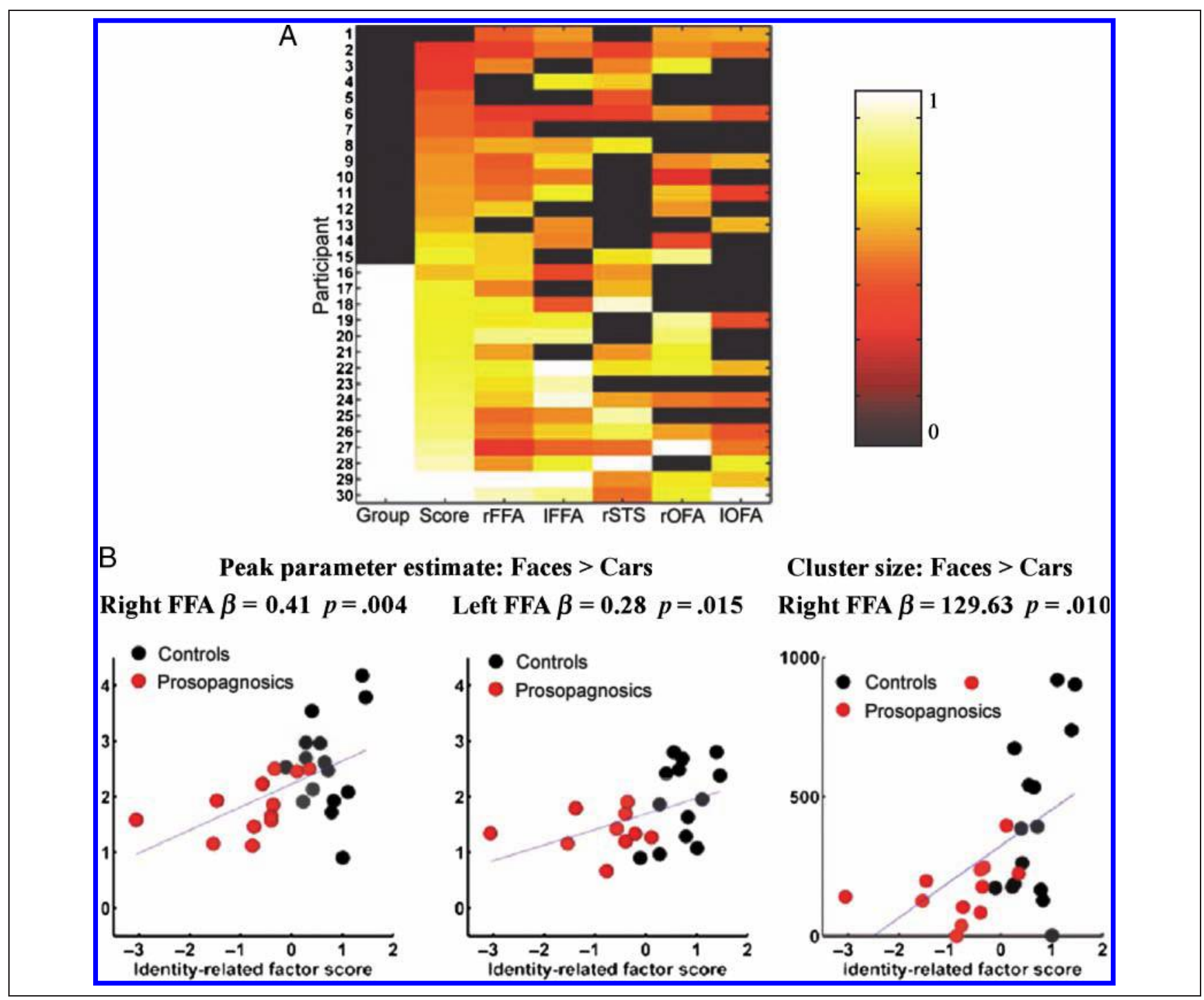

Figure 5. Face selectivity in individually defined ROIs. (A) Data are shown for ROIs defined by the contrast all faces $>$ all cars. Within each group (DPs and non-DP controls), rows are sorted by each participant's identity-related factor score. The first column represents group (black = DPs; white $=$ non-DP controls); the second is the identity-specific performance score on behavioral Factor 1 (scaled to the range 0 to 1 ). The remaining columns show face selectivity (beta parameters) for contrast all faces $>$ all cars (scaled to between 0 and 1 ). A row is filled in black when an ROI could not be identified for that individual participant. (B) The contrast (difference between beta weights) all faces $>$ all cars was computed at the fixed effects level for right (left) and left (center) individually defined FFA and then plotted here as a function of identity-related factor score, with each point representing one participant. The regression lines (fit to the entire sample) are shown in blue. Participants lacking ROIs (filled in black in Figure 5A) were not included in this analysis. The rightmost graph shows the cluster sizes (in numbers of voxels) of the right FFA, plotted against the identity-related factor score. For this analysis, all participants were included except two (see Results for details).

number of contiguous face-selective voxels) was linearly associated with the face identification factor score. In right FFA only (Figure 5), we found a significant regression using the identity-related behavioral Factor 1 scores, $\beta=$ $129.63, t(24)=2.51, p=.010$. Moreover, we observed significantly larger clusters for right FFA in non-DP controls than DPs, $t(26)=1.89, p=.003$. The correlation between cluster size in right FFA and the behavioral identification Factor 1 scores (partialling out Factors 2 and 3 ) was also significant $\rho=0.46, p=.01$. None of our face selectivity ROI measures (peak or cluster size) showed effects in right posterior STS or the OFAs.
In all our ROI regressions, we included the scores for Factors 2 and 3 (object- and expression-related factors) as regressors. These regressors were not significant, although we observed robust positive slopes and correlations for our (orthogonally derived) identification Factor 1 in bilateral FFA. Notably, this pattern of results was convergent across all our analyses of right FG or FFA activity (SPM group analysis and ROI group analysis of peaks and cluster sizes) and likewise for the temporal pole. Indeed, the numeric difference in slope between the first factor and the others was visibly striking in right FFA for both peaks and cluster sizes (Supplementary Figure 1). Although these differences 
appeared large and replicated across several convergent analyses, we did not have enough statistical power to detect interactions between one factor versus the other in relation to the fMRI data (Supplementary Table 4). Our results overall seemed consistent with the conclusion that there exists a positive linear relationship between our fMRI face selectivity measures and behavioral Factor 1, but we could offer no evidence favoring any such relationships for Factors 2 or 3 other than the relationship of Factor 3 to the amygdala and visual cortex in the SPM analysis.

\section{SPM Group Analysis of Repetition Suppression}

We evaluated identity and expression repetition suppression for each voxel in the brain (SPM group analysis) and also for individually defined ROIs. For both types of analysis, we tested identity suppression by computing the onetailed main effect of identity repetition (identity different $>$ identity same). This comparison contrasts the rows in Figure 2C: (IdEd + IdEs) > (IsEd + IsEs); see also Winston et al. (2004). Similarly, expression suppression was tested by computing the one-tailed main effect of expression repetition (expression different $>$ expressions same), which entails contrasting the columns of Figure 2C: (IdEd + IsEd) $>$ (IdEs + IsEs). We also tested for the two-tailed interaction of identity and expression suppression (IdEs $>$ IsEd) versus (IdEd > IsEs), which would reveal regions for which one type of suppression was modulated by whether the other category was same or different. On the basis of previous literature (Fox et al., 2009; Rotshtein et al., 2005; Yovel \& Kanwisher, 2005; Andrews \& Ewbank, 2004), we had expected that FG may show identity suppression whereas right (posterior and/or mid) STS would show expression suppression and that there would be no interaction effect.

We began by using SPM group analysis to test whether data from our non-DP participants replicates previous findings (Fox et al., 2009; Winston et al., 2004). Both identity and expression suppression contrasts yielded a right medial FG cluster (Figure 6), sharing the same peak voxel (MNI: $38-44-24$ ). This region overlaps with the faceselective activation seen in non-DPs (cf. Figure 3) but peaks anterior and medial to the peak showing correlations between face selectivity and identity-related behavioral factor scores (cf. Figure 4). The expression suppression contrast also showed a region (Figure 6) in right mid-STS (MNI: 52 -304 ), anterior to the peak right posterior STS face selectivity found in non-DPs (cf. Figure 4A). This mid-STS location replicates that observed in previous studies (Fox et al., 2009; Winston et al., 2004). Some studies have also reported identity suppression in the posterior right STS (Fox et al., 2009; Winston et al., 2004), but we did not observe this.

We tested whether this pattern of repetition suppression was present in DP participants. The SPM group results showed expression suppression in the right FG region.
However, the FG region was absent when we tested the identity suppression contrast. Also unlike non-DPs, there was no significant expression suppression in right midSTS. Despite these apparent differences and the overall weaker effects observed in DPs, we did not find any significant effects when testing group differences or regression models using behavioral factor scores for the repetition suppression effects. No interaction was found between identity and expression repetition in non-DPs or DPs. The regions discussed above showed no repetition suppression to cars, neither in non-DP nor DPs.

\section{Repetition Suppression in Individually Defined ROIs}

We next analyzed the peak voxel in individually defined face-selective ROIs for repetition suppression effects (Figures 7 and 8 and Supplementary Table 2), in case they might provide a more sensitive assessment for any group differences. In agreement with the SPM group analysis, non-DP participants showed significant effects in their right FFA ROIs (Figure 7) for both identity, $t(14)=4.37, p<.001$, and expression repetition suppression, $t(14)=3.59, p=$ .002 . We also found significant effects in left FFA for identity, $t(12)=3.01, p=.006$, and expression repetition suppression, $t(12)=2.19, p=.023$. There was a nearly significant trend toward expression repetition suppression in right posterior STS, $t(12)=1.60, p=.068$, but no identity suppression. We note that the SPM group analysis had localized expression repetition suppression effects to a mid-STS region anterior to the face-selective individual peaks in posterior STS (Figure 6). Therefore, the ROI group analysis (now using those latter peaks) may have reduced power for detecting these mid-STS expression effects, which appeared (according to SPM) located anterior to the ROI peaks. Interestingly, we also detected (unpredicted) expression repetition suppression in the individual right OFA ROIs (Figure 8$), t(8)=2.93, p<.010$, with some concordant nonsignificant tendency in the same direction for the left OFA, $t(7)=1.52, p=.09$.

Finally we tested whether DPs could replicate the repetition suppression pattern observed in non-DP controls. The pattern of results was similar to those of non-DPs in showing right FFA suppression for repeated identities, $t(11)=$ $3.47, p=.003$, and expressions, $t(11)=3.91, p=.001$. The left FFA in DPs showed significant expression repetition suppression, $t(9)=2.99, p=.008$, but no effect of identity repetition. As with the SPM group analysis, DPs showed no effects or trends in right posterior STS. Similarly to non-DPs, DPs showed significant expression repetition suppression in right OFA, $t(9)=4.83, p<.001$, and left OFA, $t(5)=3.04, p<.014$. The ROI group analysis of repetition suppression thus did not provide a different outcome. In line with the SPM group results, we found the overall pattern of repetition effects to be weaker in DPs compared with non-DPs, yet but without any overall significant group differences or behavioral correlations in ROIs, 
Figure 6. Identity and expression repetition suppression: SPM randomeffects analysis. (A) Regions in non-DP control participants showing significant identity repetition suppression in the right FG (left) and expression repetition suppression in right FG (middle) and right mid-STS (right). The right side of each transverse image represents the right hemisphere. (B) Pattern of mean "adjusted responses" (condition-wise beta weights, adjusted for other regressors in general linear model) in non-DP control participants from the peak right FG and right mid-STS voxels found in both identity and expression repetition suppression contrasts. (C) For comparison, we show the pattern of adjusted responses in DPs using the peak right FG and mid-STS voxels shown in (B). All participants were included in SPM analyses.

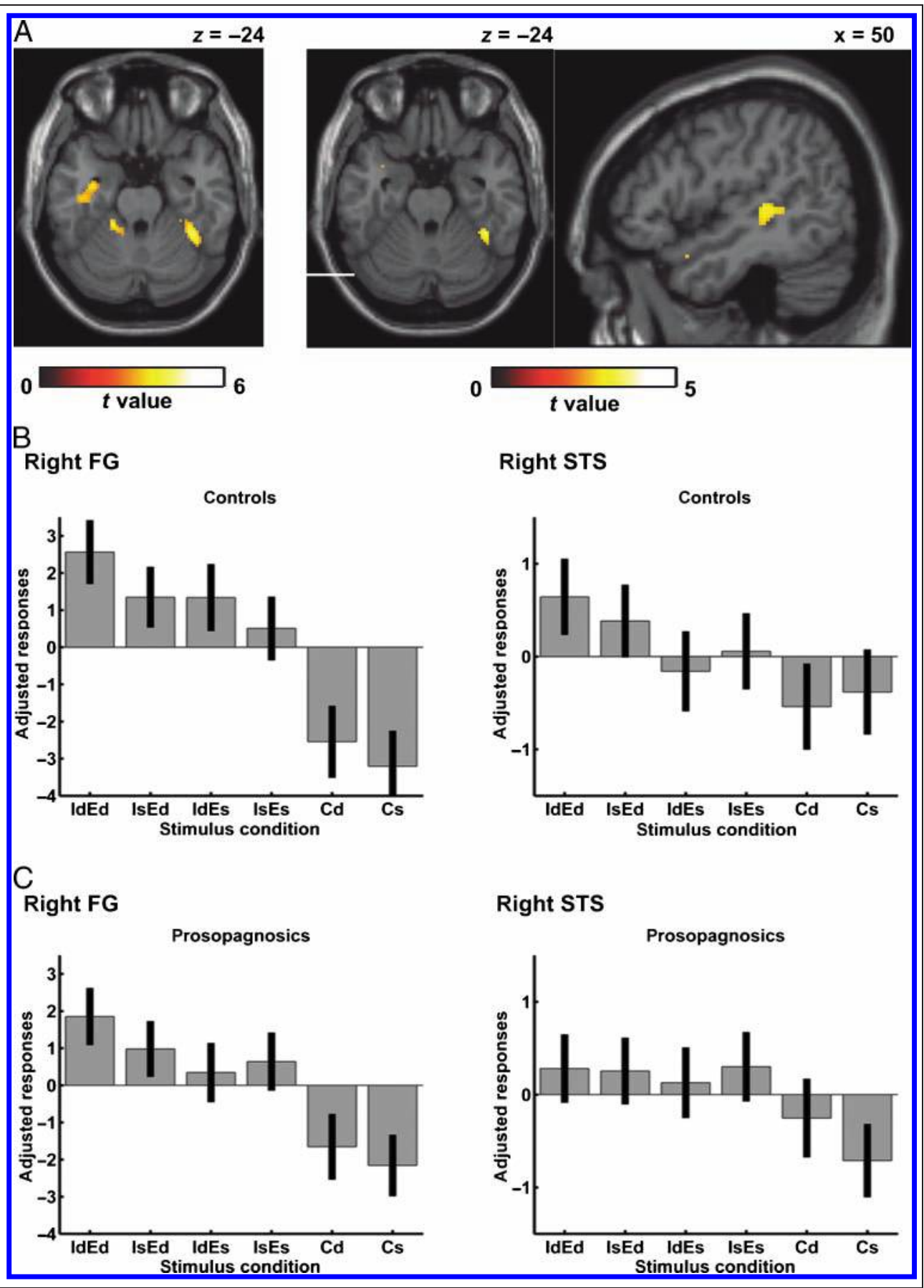

for either identity or expression repetition suppression. Also in agreement with the SPM group analysis, we detected no interactions between identity and expression suppression in any ROI, either for non-DPs or for DPs. Car repetition did not show any effects in any ROI, either for non-DPs or for DPs.

In summary, our measures of identity- and expressionspecific repetition suppression replicated the expected findings in non-DP participants. FG showed suppression to repeated expressions and identities, whereas right midSTS showed suppression to expressions. However, this did not differ significantly between groups (although repetition suppression tended to be weaker in DPs) nor did it relate significantly to the behavioral factor scores.

This contrasts with the robust statistical relationships we had observed between face selectivity per se (faces $>$ cars) in FG (or individually defined FFA) with the behavioral Factor 1 of identity-related recognition ability. That effect was observed for multiple measures of face selectivity, including face selectivity at individual voxels in SPM group analysis, at the peak face selectivity of ROIs functionally defined in individuals, and the anatomic extent 
of the face selectivity in these ROIs. Our results thus provide new evidence that the neural mechanisms expressing face selectivity do relate to individual differences in face identification performance, although repetition suppression effects apparently may not.

\section{DISCUSSION}

We explored the relationships between behavioral face recognition ability and two fMRI measures of neural processing for faces (face selectivity and repetition suppression), exploiting the wide range of face identification performance among 15 DPs and 15 matched non-DP participants. As a group, DPs showed reduced face-selective responses in the bilateral FFA and smaller face-selective clusters in right FFA. We employed a factor analytic approach to our behavioral battery, as recently reported in our related structural MR study (Garrido et al., 2009). For this approach, we derived behavioral scores from standard factor analysis of all participants' performance on the test battery. This yielded three orthogonal components of behavioral variability related to recognition for facial identity, nonface objects, and facial expressions.

Our analyses demonstrate that the identity-related behavioral factor was linearly related (when considering every individual in our sample, in accord with our "individual differences" approach) to fMRI face selectivity in the left anterior temporal lobe and right FG. This effect was observed for FG face selectivity as identified in SPM group analysis and for the peaks and anatomic extent of individually defined FFA ROIs. These results demonstrate that better performance in facial identity processing tasks was positively associated with fMRI face selectivity in FG bilaterally and left anterior temporal lobe, with peak face selectivity in the right and left individually defined FFA, and with the size of the right FFA. The significant brain-behavior relations we observed applied only to the behavioral component relating to face identification performance (but see Supplementary Table 4), even when controlling for the other two factors (related to performance for facial expressions or nonface objects). The second and the third factors were not related to any of our three face selectivity fMRI measures: SPM voxels, ROI peaks, or ROI cluster size. The absence of face-selective ROIs, including for right posterior STS, was also associated with DP-related deficits. Thus, we provide abundant new evidence linking face identification ability to individual differences in function for core face-selective areas (FG, posterior STS) in relation to fMRI face selectivity (assessed here by the face $>$ car contrast). Although we found several forms of repetition suppression in these and related regions, the repetition
Figure 7. Identity and expression repetition suppression in individually defined FFA. Average peak parameter estimate (beta weight) with 95\% confidence intervals in right and left for the six conditions. Also shown are $p$ values less than .05 for identity repetition suppression (denoted "identity") and expression repetition suppression (denoted "expression").

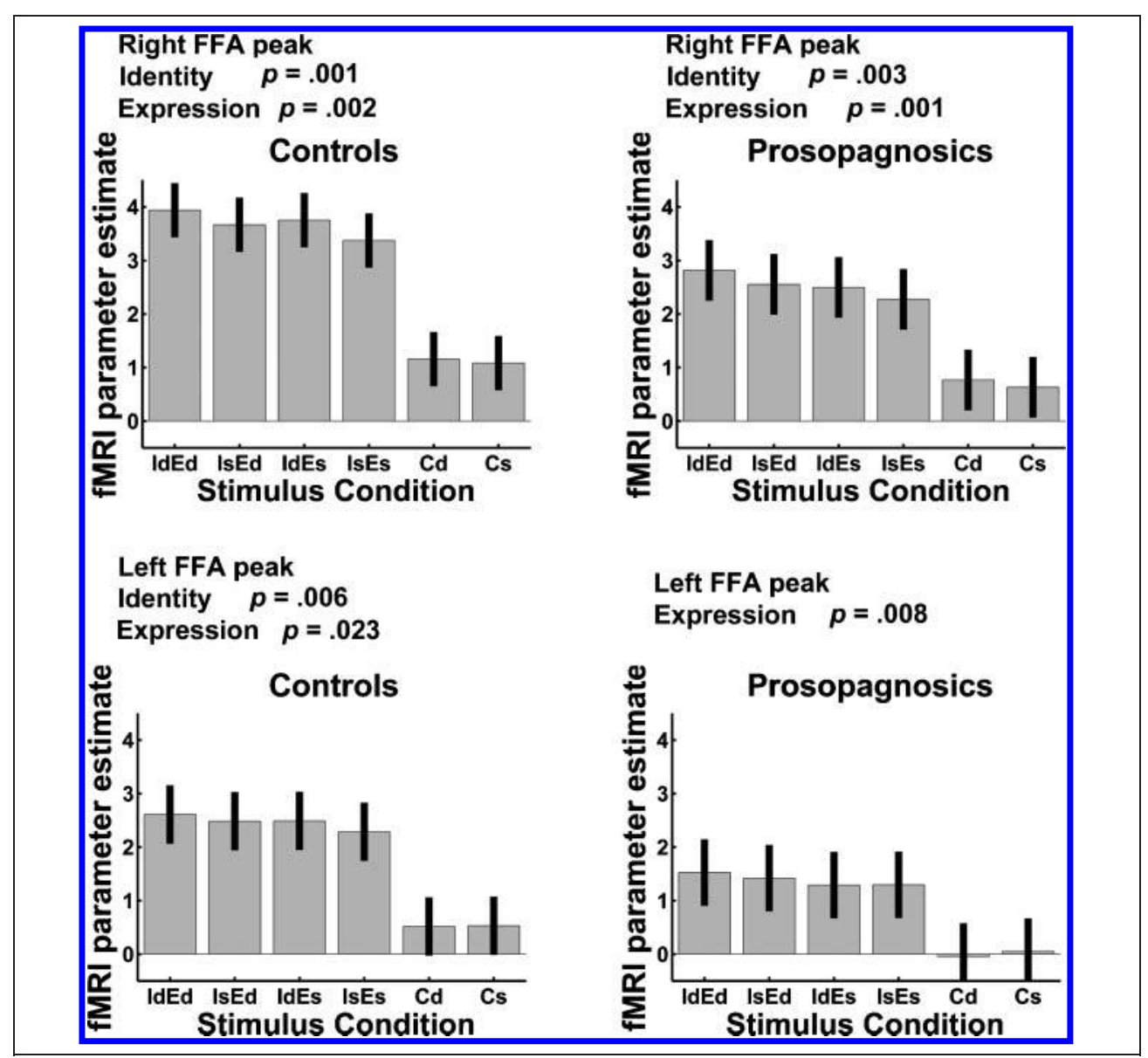


Figure 8. ROI identity and expression repetition suppression in posterior STS and OFA. Average peak parameter estimate (beta weight) with $95 \%$ confidence intervals in right and left OFA plus right posterior STS for the six conditions. Also shown are significant and near-significant $p$ values for identity repetition suppression (denoted "identity") and expression repetition suppression (denoted "expression").

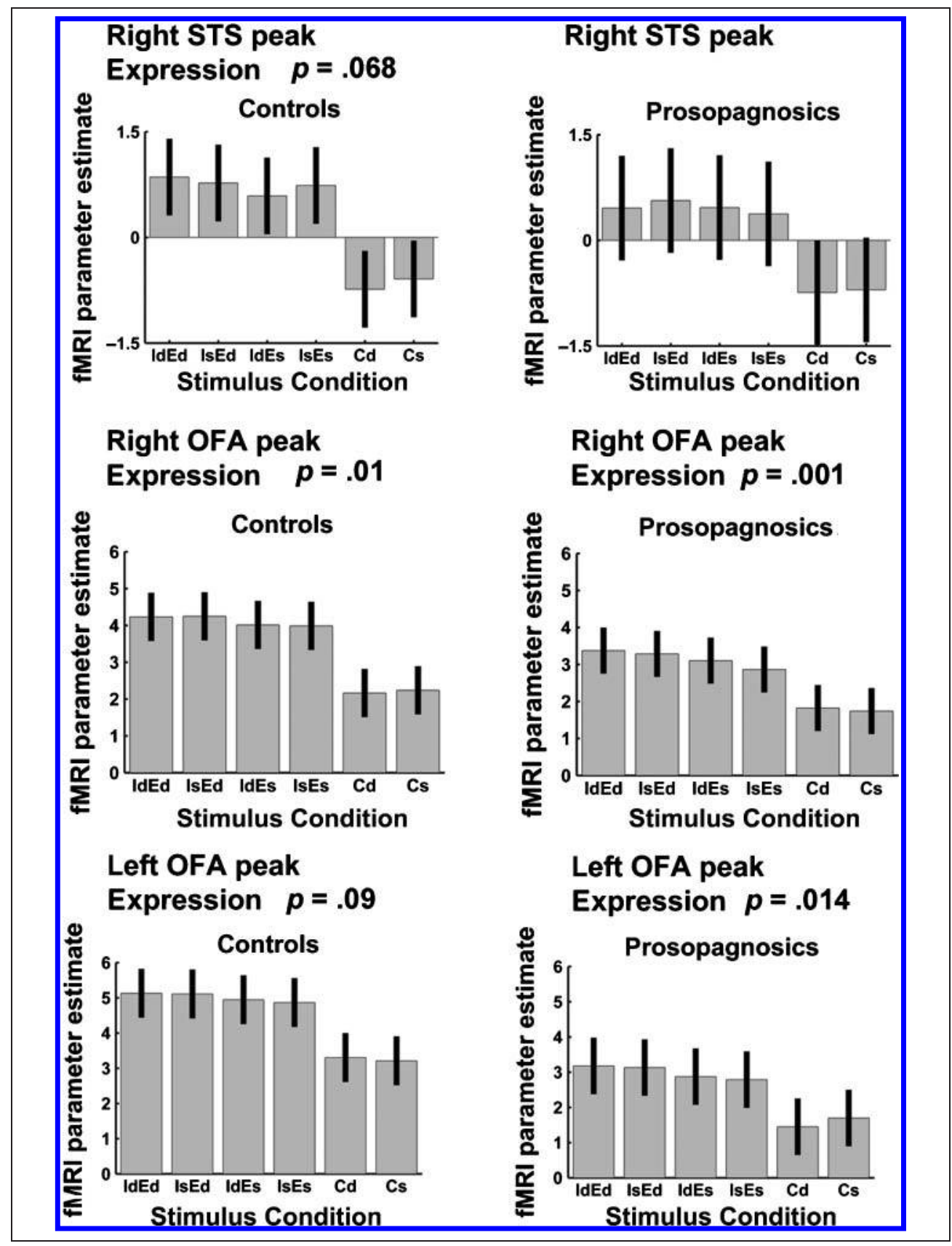

phenomena were not strongly linked to individual differences in behavior, unlike the face selectivity per se.

\section{Face Selectivity and Successful Recognition of Identities}

The FG and the FFA have received considerable research attention. Its robust face selectivity across many normal observers suggests that it may provide an important mechanism for face representation. More controversially, it has been claimed that FG and/or FFA may provide specialized mechanisms for individuating identities as opposed to recognition of other physical attributes of faces (Rotshtein et al., 2005; Haxby et al., 2000). Such claims have led to the prediction that behavioral success in differentiating identities (and individual differences in this ability) may be correlated with FG face selectivity. Our new data in relation to individual differences in face identification skill (across non-DP and DP participants considered as one sample of variation in such skill) provide new evidence consistent with this view.

Our approach allowed us to detect quantitative relationships between fMRI measures and the magnitude of behavioral skill. A quantitative (rather than all-or-none) relationship between the extent of face identification ability and face selectivity may, in part, help explain the heterogeneous results seen in the previously published DP case studies, typically examining a smaller number of participants 
(Minnebusch et al., 2009; Bentin et al., 2007; Williams et al., 2007; Avidan et al., 2005; Hasson et al., 2003; Hadjikhani \& de Gelder, 2002). In our study, nearly all DPs (12 of 15 DPs) evinced normal-appearing right FFAs when we inspected cases individually. We observed more quantitative relationships here, which may be consistent with DP individuals forming the tail end of a potentially more continuous distribution of facial recognition ability. Such continuous variability might also potentially explain why the SPM group analysis did not reveal significant effects in FG for categorical comparisons between group means. Significant FG findings were instead most apparent for regressions aimed at detecting continuous linear relationships rather than discrete differences. Although our ROI group analysis of peak selectivity and cluster size was sensitive to some discrete differences, our data suggest that many DPs may be part of the wider spectrum of face identification ability, although this "continuum" versus "dichotomy" issue requires further research with even larger samples.

Beyond FG/FFA, our data implicate additional regions in face identification ability. Right posterior STS face selectivity was absent or abnormal in many DPs (Figure 5), although we did not find statistically robust regression effects for the face identification factor in this region (but note that there were fewer participants in the analysis for STS ROIs). Intriguingly, right STS was also implicated in our previous study of the structural correlates of DPrelated face impairments, using the same participant population (Garrido et al., 2009). In that study, a mid-STS region (somewhat anterior to the more posterior STS face selectivity we observed here) showed decreased gray matter volume in our DP population compared with the same matched non-DP control sample.

It is notable also that the SPM group analysis revealed a sizable correlation of fMRI face selectivity with behavioral face identification near the left temporal pole (Table 2). Previous fMRI work suggests that (in non-DP participants) anterior temporal cortex may subserve identity-specific face perception (Rotshtein et al., 2005) and may represent person-specific conceptual knowledge (Simmons \& Martin, 2009). At least in the right hemisphere, anterior temporal cortex further can signal information about individual identities (Kriegeskorte, Formisano, Sorger, \& Goebel, 2007). Moreover, for DPs, there are recent reports of some structural abnormalities in temporal pole (Behrmann et al., 2007). Our previous structural study showed a similar temporal pole effect in the same sample of DPs we report here, although in the right hemisphere (Garrido et al., 2009). This further accords with recent findings that the white matter integrity of connections between regions in the occipital and posterior temporal lobes and more anterior temporal regions are reduced in some DPs, as suggested recently using diffusion tensor tractography (Thomas et al., 2009). Interestingly, a DP case study (von Kriegstein, Kleinschmidt, \& Giraud, 2006) seems to accord with such an interregional perspective by showing not only reduced face responses in the anterior temporal lobe but also re- duced functional connectivity between right FG and left anterior lobe compared with a non-DP control population.

Although these anterior temporal regions may be structurally deficient in DPs, Behrmann et al. (2007) did not find structural or functional deficits that overlapped with core functional regions (such as posterior or mid-FG). In contrast, our previous structural study with the same participants as reported here (Garrido et al., 2009) revealed reduced gray matter in DPs for a mid-FG region, which was relatively near to our face-selective FG region here. To provide an initial exploration into possible relations between structural MR outcomes and functional MR outcomes within the same sample, we used our previously published structural measures (see Garrido et al., 2009) to explore the idea that reduced functional face selectivity (in fMRI) might relate to deficient underlying gray matter in structural MR (Supplementary Results and Supplementary Table 4). However, this preliminary analysis found no significant correlations between functional and structural measures for any of the ROIs showing face selectivity reductions with worse face identification here. Future research with larger samples will be needed to unravel the structural-functional relation in the context of face identification skills. Moreover, this may require sophisticated multivariate analysis approaches because it is possible that structural variation in one (or several) particular brain region could lead to functional variation in remote but interconnected regions. Hence, those areas showing structural variation may not have a one-to-one correspondence with those showing functional variation.

\section{Measuring Orthogonal Components of Recognition Ability}

We included several tasks (including the CFMT and the FFT) that putatively measure face identification ability. Performance on any one of these tasks, taken by itself, is presumably attributable in part to identity-related processing. Yet any raw putative identity-related measure (taken by itself) may be potentially confounded by variability attributable to other visual processes also or to the participant's attention during that task. The factor analysis method can provide some greater assurance that the variability associated with the emergent identity-related performance factor is related only to that portion of variability held in common by a diverse range of face identification-related tasks. Moreover, the factor analysis also ensures this variability will be orthogonal to that associated with other factors, as for the object and expression components here.

Despite the effectiveness of this factor analytic approach, we believe that it could be further improved. A greater number and diversity of behavioral measures and participants will presumably produce increasingly "pure," interpretable, and useful resultant factors. For example, our face identification factor here related to several memory tests (e.g., CFMT) and so might be rendered more identity specific by including more perception-based tests. It is reassuring in 
this respect that the identity-matching test loaded highly on our Factor 1, despite minimal demands on long-term memory. However, it is interesting that another perceptual test, CFPT, did not load as highly. Indeed, the factor analysis partitioned variability in CFPT performance not only into that held in common with other facial identification tasks but also (separately) into some CFPT variability held more in common with object tasks. This finding underscores the potential advantage of factor analysis over reporting relationships with raw measures alone, as the latter approach could potentially mix variability related to different abilities that jointly contribute to any single task.

We acknowledge also that our power for detecting relationships with object- and expression-related factor scores here may have been more limited than for face identification factor scores because of restricted ranges (compared with the identity-related factor). We were specifically interested here in detecting correlations related to identity recognition, so we recruited individuals (DPs) deficient in identity processing in addition to the non-DP participants. The resulting sample may not include individuals varying to the same degree on object or expression recognition. This could be addressed in future work by specifically sampling also (or instead) individuals with marked object or expression deficits. Despite the potential range restriction, we nevertheless already sampled sufficient variability to detect some brain-behavior relationships not related to facial identity. In particular, we note our finding that amygdala face selectivity related to the expression factor score, a finding consistent with numerous studies showing amygdala involvement in facial expression and emotion representation (e.g., Das et al., 2005; Vuilleumier et al., 2003).

\section{Repetition Suppression}

In addition to investigating face selectivity, we were also interested in repetition suppression as a further measure potentially relevant to facial identity representations. Our results in non-DP participants are similar to previous results from other studies and appear largely consistent with the model of Haxby et al. (2000). As expected, we found that identity repetition leads to reduced responses in right mid-FG; expression repetition leads to reduced fMRI responses in right STS, whereas no interaction effect was found between the two types of repetition (see also Winston et al., 2004). We further detected expression repetition effects in right mid-FG (see also Fox et al., 2009). Our findings of both identity and expression repetition suppression in FG may bear on whether anatomically separable or functionally independent pathways subserve identity versus expression processing, potentially favoring the alternative proposal that form-based representations of identities and expressions may share some common visual representations (Calder \& Young, 2005).

These suppression effects were also expressed in DPs, albeit more weakly. Thus, our results further replicated other previous findings that did not show clear repetition suppression anomalies in DPs (Avidan \& Behrmann, 2009; Avidan et al., 2005). The absence of a group difference for repetition suppression and, in particular, of any correlation for such suppression with the face identification behavioral factor contrasts with the positive results obtained using face selectivity fMRI measures. This difference between two measures of face-related neural processing deserves further exploration, as it raises interesting questions about the extent to which face selectivity and repetition suppression may reflect the same or different processes. Although many studies a priori assumed repetition suppression to be a conclusive marker for neural coding of the repeated information, in fact the mechanisms underlying repetition suppression are not well understood (Grill-Spector, Henson, \& Martin, 2006), complicating direct interpretation of their results. A recent study (Mur, Ruff, Bodurka, Bandettini, \& Kriegeskorte, 2010) suggests that identity suppression may not always reflect neural coding of identity but could potentially result from a variety of other causes including declining attention. Moreover, abnormal repetition suppression can arise despite intact face selectivity, in some cases of acquired prosopagnosia with lesions in the vicinity of OFA (Steeves et al., 2009; Schiltz et al., 2006).

One testable possibility is that repetition suppression reflects representations that support different behaviors than face selectivity. For example, repetition suppression may better relate to repetition-related behavioral effects such as priming (which were not examined behaviorally here), whereas face selectivity might support more explicit recognition judgments, like those made here. Further studies could go on to test whether individual differences in repetition suppression are associated with more implicit measures of face processing. Given that repetition suppression effects in fMRI experiments can be much smaller than face selectivity effects, magneto-encephalography might provide more comparably sized measures of face selectivity and repetition suppression (for large repetition suppression effects in magneto-encephalography, see Furl, van Rijsbergen, Treves, Friston, \& Dolan, 2007; Harris \& Nakayama, 2006). Repetition suppression measures could also be compared against other representational markers, such as multivariate pattern decoding, in the future (e.g., Kriegeskorte et al., 2007).

Finally, we found that identity repetition suppressed FG activity, despite variations in expression, viewpoint, and other low-level image features across the repeated identities here. Although this result contrasts with some previous studies that did not report clear identity-specific repetition effects in FG (Davies-Thompson, Gouws, \& Andrews, 2009; Pourtois, Schwartz, Seghier, Lazeyras, \& Vuilleumier, 2005a, 2005b; Andrews \& Ewbank, 2004), there may be some limited viewpoint dependence (Ewbank \& Andrews, 2008). Pourtois et al. (2005b) found limited generalizability of identity-specific suppression between three-quarter view and frontal faces (as we used) in FG, whereas Ewbank and Andrews (2008) showed that generalization declined with increasing change in viewpoint angle. 


\section{Conclusions}

Face selectivity and repetition suppression in core face processing regions have become increasingly accepted as neural measures for face processing. Previous studies of DP had typically not found definitive evidence for reduced face selectivity or repetition suppression in these core areas. To address this issue, we scanned 15 DPs and 15 matched non-DP controls in conjunction with a factor analytic method for deriving identity-related summary behavioral scores from a battery of tests (as opposed to expression or nonface object relates scores). Although we found no compelling relationship between these behavioral scores and repetition suppression, we found clear evidence that fusiform face selectivity in fMRI relates systematically to individual differences in behavioral face identification ability. This was found across voxel-wise SPM group analysis, for the peaks of individually defined FFAs, and for the anatomic extent of the individual FFAs. No such relationship was found between FG/FFA activity or size with object or expression recognition ability. These results indicate that fusiform face selectivity provides a marker for neural mechanisms that determine variation in face identification ability.

\section{Acknowledgments}

The authors are grateful to their colleagues at the University College London, namely, Laura Germine and Raka Tavashmi for assistance with behavioral measurement and/or fMRI data collection, Bogdan Draganski and Ferath Kherif for advice and support, and John Stevens for clinical evaluation of the participants. This work was supported by the Portuguese Foundation for Science and Technology (grant no. SFRH/BD/22580/2005 to L. G.), the Economic and Social Research Council (grant no. RES-061-230400 to B. D.), and The Wellcome Trust (R. J. D. and J. D.). J. D. is a Royal Society Research Professor. Editorial assistance for the manuscript was provided by the National Institutes of Health Fellows Editorial Board.

Reprint requests should be sent to Nicholas Furl, National Institutes of Health, Building 49, 1B80, 49 Convent Dr., Bethesda, MD, 20892, or via e-mail: furlno@mail.nih.gov.

\section{REFERENCES}

Allison, T., Puce, A., \& McCarthy, G. (2000). Social perception from visual cues: Role of the STS region. Trends in Cognitive Sciences, 4, 267-278.

Andrews, T. J., \& Ewbank, M. P. (2004). Distinct representations for facial identity and changeable aspects of faces in the human temporal lobe. Neuroimage, 23, 905-913.

Avidan, G., \& Behrmann, M. (2009). Functional MRI reveals compromised neural integrity of the face processing network in congenital prosopagnosia. Current Biology, 19, 1146-1150.

Avidan, G., Hasson, U., Malach, R., \& Behrmann, M. (2005). Detailed exploration of face-related processing in congenital prosopagnosia: II. Functional neuroimaging findings. Journal of Cognitive Neuroscience, 17, 1150-1167.

Baron-Cohen, S., Wheelwright, S., Hill, J., Raste, Y., \& Plumb, I. (2001). The "Reading the Mind in the Eyes" test revised version: A study with normal adults, and adults with Asperger syndrome or high-functioning autism. Iournal of Child Psychology and Psychiatry, 42, 241-251.

Behrmann, M., Avidan, G., Gao, F., \& Black, S. (2007). Structural imaging reveals anatomical alterations in inferotemporal cortex in congenital prosopagnosia. Cerebral Cortex, 17 , 2354-2363.

Behrmann, M., Avidan, G., Marotta, J. J., \& Kimchi, R. (2005). Detailed exploration of face-related processing in congenital prosopagnosia: I. Behavioral findings. Journal of Cognitive Neuroscience, 17, 1130-1149.

Bentin, S., Degutis, J. M., D'Esposito, M., \& Robertson, L. C. (2007). Too many trees to see the forest: Performance, event-related potential, and functional magnetic resonance imaging manifestations of integrative congenital prosopagnosia. Journal of Cognitive Neuroscience, 19, 132-146.

Calder, A. J., \& Young, A. W. (2005). Understanding the recognition of facial identity and expression. Nature Reviews Neuroscience, 6, 641-651.

Crawford, J. R., \& Howell, D. C. (1998). Comparing an individual's test score against norms derived from small samples. Clinical Neuropsychology, 12, 482-486.

Das, P., Kemp, A. H., Liddell, B. J., Brown, K. J., Olivieri, G., Peduto, A., et al. (2005). Pathways for fear perception: Modulation of amygdala activity by thalamocortical systems. Neuroimage, 26, 141-148.

Davies-Thompson, J., Gouws, A., \& Andrews, T. J. (2009). An image-dependent representation of familiar and unfamiliar faces in the human ventral stream. Neurobsvchologia, 47, 1627-1635.

Duchaine, B., Germine, L., \& Nakayama, K. (2007). Family resemblance: Ten family members with prosopagnosia and within-class object agnosia. Cognitive Neuropsychology, 24, 419-430.

Duchaine, B., \& Nakayama, K. (2005). Dissociations of face and object recognition in developmental prosopagnosia. Journal of Cognitive Neuroscience, 17, 249-261.

Duchaine, B., \& Nakayama, K. (2006a). The Cambridge Face Memory Test: Results for neurologically intact individuals and an investigation of its validity using inverted face stimuli and prosopagnosic participants. Neuropsychologia. 44, 576-585.

Duchaine, B. C., \& Nakayama, K. (2006b). Developmental prosopagnosia: A window to content-specific face processing. Current Opinion in Neurobiology, 16, 166-173.

Duchaine, B., Yovel, G., \& Nakayama, K. (2007). No global processing deficit in the Navon task in 14 developmental prosopagnosics. Social Cognitive and Affective Neuroscience, 2, 104-113.

Ewbank, M. P., \& Andrews, T. J. (2008). Differential sensitivity for viewpoint between familiar and unfamiliar faces in human visual cortex. Neuroimage, 40, 1857-1870.

Fox, C. J., Moon, S. Y., Iaria, G., \& Barton, J. J. (2009). The correlates of subjective perception of identity and expression in the face network: An fMRI adaptation study. Neuroimage, 44, 569-580.

Furl, N., van Rijsbergen, N. J., Treves, A., Friston, K. J., \& Dolan, R. J. (2007). Experience-dependent coding of facial expression in superior temporal sulcus. Proceedings of the National Academy of Sciences. U.S.A., 104, 13485-13489.

Garrido, L., Duchaine, B., \& Nakayama, K. (2008). Face detection in normal and prosopagnosic individuals. Journal of Neuroscience, 2, 219-240.

Garrido, L., Furl, N., Draganski, B., Weiskopf, N., Stevens, J., Tan, G., et al. (2009). Voxel-based morphometry reveals reduced grey matter volume in the temporal cortex of developmental prosopagnosics. Brain, 132, 3443-3455. 
Grill-Spector, K., Henson, R., \& Martin, A. (2006). Repetition and the brain: Neural models of stimulus-specific effects. Trends in Cognitive Sciences, 10, 14-23.

Hadjikhani, N., \& de Gelder, B. (2002). Neural basis of prosopagnosia: An fMRI study. Human Brain Madping. 16, $176-182$

Harris, A., \& Nakayama, K. (2006). Rapid face-selective adaptation of an early extrastriate component in MEG Cerebral Cortex, 17, 63-70.

Hasson, U., Avidan, G., Deouell, L. Y., Bentin, S., \& Malach, R. (2003). Face-selective activation in a congenital prosopagnosic subject. Iournal of Cognitive Neuroscience, 15, 419-431.

Haxby, J. V., Hoffman, E. A., \& Gobbini, M. I. (2000). The distributed human neural system for face perception. Trends in Cognitive Sciences, 4, 223-233.

Hein, G., \& Knight, R. T. (2008). Superior temporal sulcus-It's my area: Or is it? Journal of Cognitive Neuroscience, 20, 2125-2136.

Humphreys, K., Avidan, G., \& Behrmann, M. (2007). A detailed investigation of facial expression processing in congenital prosopagnosia as compared to acquired prosopagnosia. Experimental Brain Research, 176, 356-373.

Kriegeskorte, N., Formisano, E., Sorger, B., \& Goebel, R. (2007). Individual faces elicit distinct response patterns in human anterior temporal cortex. Proceedings of the National Academv of Sciences. U.S.A., 104, 20600-20605.

Lundqvist, D., Flykt, A., \& Öhman, A. (1998). The Karolinska directed emotional faces-KDEF [CD-ROM]. Stockholm, Sweden: Department of Clinical Neuroscience, Psychology Section, Karolinska Institutet.

Minnebusch, D. A., Suchan, B., Köster, O., \& Daum, I. (2009). A bilateral occipitotemporal network mediates face perception. Behavioural Brain Research, 198, 179-185.

Mur, M., Ruff, D. A., Bodurka, J., Bandettini, P. A., \& Kriegeskorte, N. (2010). Face-identity change activation outside the face system: "Release from adaptation" may not always indicate neuronal selectivity. Cerebral Cortex, doi:10.1093/cercor/bhp272.

Pourtois, G., Schwartz, S., Seghier, M. L., Lazeyras, F., \& Vuilleumier, P. (2005a). Portraits or people? Distinct representations of face identity in the human visual cortex. Journal of Cognitive Neuroscience, 17, 1043-1057.

Pourtois, G., Schwartz, S., Seghier, M. L., Lazeyras, F., \& Vuilleumier, P. (2005b). View-independent coding of face identity in frontal and temporal cortices is modulated by familiarity: An event-related fMRI study. Neuroimage, 24, 1214-1224.
Rotshtein, P., Henson, R. N., Treves, A., Driver, J., \& Dolan, R. J. (2005). Morphing Marilyn into Maggie dissociates physical and identity face representations in the brain. Nature Neuroscience, 8, 107-113.

Schiltz, C., Sorger, B., Caldara, R., Ahmed, F., Mayer, E., Goebel, R., et al. (2006). Impaired face discrimination in acquired prosopagnosia is associated with abnormal response to individual faces in the right middle fusiform gyrus. Cerebral Cortex, 16, 574-586.

Simmons, W. K., \& Martin, A. (2009). The anterior temporal lobes and the functional architecture of semantic memory. Iournal of the International Neuropsychological Societv. 15, 645-649.

Steeves, J., Dricot, L., Goltz, H. C., Sorger, B., Peters, J., Milner, A. D., et al. (2009). Abnormal face identity coding in the middle fusiform gyrus of two brain-damaged prosopagnosic patients. Neuropsvchologia. 47, 2584-2592.

Thomas, C., Avidan, G., Humphreys, K., Jung, K. J., Gao, F., \& Behrmann, M. (2009). Reduced structural connectivity in ventral visual cortex in congenital prosopagnosia. Nature Neuroscience, 12, 19-31.

van de Stock, J., van de Riet, W. A., Righart, R., \& de Gelder, B. (2009). Neural correlates of perceiving emotional faces and bodies in developmental prosopagnosia: An event-related fMRI-study. PLOS ONE, 3, e3195.

von Kriegstein, K., Dogan, O., Grüter, M., Giraud, A. L., Kell, C. A., Grüter, T., et al. (2008). Simulation of talking faces in the human brain improves auditory speech recognition. Proceedings of the National Academv of Sciences, U.S.A. 105, 6747-6752.

von Kriegstein, K., Kleinschmidt, A., \& Giraud, A. L. (2006). Voice recognition and cross-modal responses to familiar speakers' voices in prosopagnosia. Cerebral Cortex, 16, 1314-1322.

Vuilleumier, P., Armony, J. L., Driver, J., \& Dolan, R. J. (2003). Distinct spatial frequency sensitivities for processing faces and emotional expressions. Nature Neuroscience, 6, 624-631.

Williams, M. A., Berberovic, N., \& Mattingley, J. B. (2007). Abnormal fMRI adaptation to unfamiliar faces in a case of developmental prosopamnesia. Current Biology, 17, 1259-1264.

Winston, J. S., Henson, R. N., Fine-Goulden, M. R., \& Dolan, R. J. (2004). fMRI-adaptation reveals dissociable neural representations of identity and expression in face perception. Iournal of Neuropbusiology, 92, 1830-1839.

Yovel, G., \& Kanwisher, N. (2005). The neural basis of the behavioral face-inversion effect. Current Biologv, 15, $2256-2262$. 
This article has been cited by:

1. Galia Avidan, Michal Tanzer, Marlene Behrmann. 2011. Impaired holistic processing in congenital prosopagnosia. Neuropsychologia . [CrossRef]

2. Kevin J Mitchell. 2011. Curiouser and curiouser: genetic disorders of cortical specialization. Current Opinion in Genetics \& Development . [CrossRef] 